\title{
DESIGN AND IMPLEMENTATION OF A COLLABORATIVE PLATFORM MODEL FOR EPIDEMIC AND COLLECTIVE HEALTH SERVICES
}

\author{
Lucas C. de Almeida, Daniel S. do Prado, Lucas M. C. e Martins, \\ Francisco L. de Caldas Filho, Fábio L. L. de Mendonça and Rafael T. de Sousa Jr \\ Electrical Engineering Department, University of Brasilia. Brasilia, DF, 70910-900, Brazil
}

\begin{abstract}
The aggregation and visualization of data are part of one of the most important fields of Data Science's application today. This class of tools evidences its importance in the area of public health, especially in the context of epidemics and pandemics. However, there are several issues in accessing reliable data, such as diverse sources, economic interests, and technical difficulties. This work proposes and explores the technological choices for the implementation of a system in which, collaboratively, users provide health information, which are made available through panels with scalability in access and flexibility in the analysis, allowing a macro view of the situation. Also, with the use of these visualizations and mobile application technologies, users can take advantage of services such as monitoring health status and sending alerts of emergency situations.
\end{abstract}

\section{KEYWORDS}

Epidemic Data, Data Analysis, Data Visualization, Data Science, Collaborative Health Data Systems, Collective Health

\section{INTRODUCTION}

One of the biggest problems in urban centers is to understand any phenomenon that spreads among the population in a short period of time and has the potential to reach all citizens. Among the several possible scenarios, one requires monitoring and quick adjustment of decisions: the context of an epidemic. In Lai, Yeung \& Celi (2020) and Ienca \& Vayena (2020), this issue is explored, conveying the role of technology in these scenarios. 
IADIS International Journal on WWW/Internet

This situation was verified dramatically during the COVID-19 disease pandemic. The world is in the midst of a highly contagious disease without a scientifically proven medicine at the time of this study and with only a few people that received vaccines worldwide until late December 2020 (WHO, 2020). In this scenario, WHO (2020) states that contagion prevention is the best way to avoid exposure to the virus.

Therefore, analysis of data on the location of the verified cases, notification date, and onset of symptoms, age, even the physical characteristics of those infected have proved to be extremely important for understanding the speed of spread and the risk profile for the disease. In addition, over time, certain regions began to show an infection pattern different from those around them, requiring additional measures of social distance being guaranteed by the government itself (an example can be seen in Consultor Juridico [2020]). None of these measures and decisions would make sense if there were no ways to measure impacts and make comparisons with the past. Data science visualization, aggregation and analysis systems are the most suitable tools for these studies.

However, even though the importance of technology and analysis in epidemic situations is widely known, there are still global difficulties in integrating databases, such as patient records, monitoring infected people, medical prescriptions delivered in public and private health, among others, all related to the technical difficulty in inserting structured collection methods and data cleaning in the workflow of health professionals for further analysis (in Santos et al. [2017] it is possible to see a study on the topic). Faced with this problem, the option arises to allow the end user (as opposed to health units) the power to provide data, which, when widely accepted by the community, results in a highly valuable and important database in the generation of metrics that expose the general health situation of the population. Yet, while the user willingly provides his own data, he can also easily take advantage of services that help him maintain and even improve health, such as individual protection guidelines in relation to the social distance indicated during the epidemic, contagious diseases, health monitoring services (for sick / infected people), and even generation of personal historic.

In addition, an information system of this nature also brings a great concern with the security of the data that is manipulated and made available to its users. This concern stands out in two aspects that need to be addressed: the confidence that the system has in data provided by each user and the proper presentation of data for each type of user.

In this work, a system divided into modules will be presented, which, in summary, allows users to collaboratively provide their own health data and access basic services such as emergency button and verification of individual protection guidelines. This work is an extended version the work de Almeida et al. (2020) in which the system is briefly described. The resulting database is then consumed by an application that makes it possible to analyze these data both in georeferenced visualization and in numbers and historical series. This system, made up of a programming interface, mobile application, and database management system, was put into production in a university environment and allowed the fast concept implementation of a collective health monitoring room. The objective is to show how much technology can contribute, in a simple and efficient way (as long as it is well directed), to problems as current and relevant as the population's health. Also, it is intended that the project logics used in this work are referenced and serve as a support for the elaboration of other projects with similar objectives. 
This document consists of five sections, including this introduction. The next section, number 2, deals with related works. Section 3 specifies the proposed system combining technologies that are widely used in industry and that integrate seamlessly into the context of users. Section 4 serves to discuss and demonstrate the results obtained in implementing the project with real data. The conclusions and future work can be found in Section 5.

\section{RELATED WORKS}

Important advances have been made over the years to improve the capture and analysis of data in real time via mobile applications and other techniques in the field of data mining. These advances have been reflected in consistent studies that allow us to see the practical benefits of using such technologies and methods in combating the proliferation of contagious diseases, both for diagnostic purposes and for their treatment and monitoring. Among the possible visualizations, the georeferenced representations stand out, being the main benefit of the project described in this article.

Geographic information solutions such as real-time (or almost real) online mapping of disease cases and social media reactions to the spread of information, along with predictive risk mapping, using mobile data from space and time in locomotion of individuals, are proving to be indispensable for the monitoring and timely and effective response to epidemics, as seen in the work developed by Boulos \& Geraghty (2020). The applications suggested in the referenced work aim to capture user displacement data to establish measurements and assessments of the geographical spread of contagious diseases. Comparatively, the system implemented in this project has a broader contribution potential, since it expands the aid in mapping the geographical spread of diseases to also cover individual monitoring and the detection and collection of other valuable parameters such as the health profile of users.

In the work developed by Arias-Carrasco et al. (2020), the problem of the lack of free and open tools to allow the general public to view relevant geographic data on a currently highlighted epidemic on a dashboard, or friendly panel for ordinary users, was addressed. Being used for studies or transparency before the population, this tool can be of great help in studying and fighting diseases and infections. In the referenced work, a specific panel was developed for this purpose, that is the providing simplified, intuitive, and friendly views with graphics on specific epidemics. The data that feeds the panel proposed in the aforementioned work comes from the public that will access the panel, which can impact the representativeness of the information. In a different way, in this project, in addition to a friendly dashboard, data is captured directly from users through an application with standardized notification format. Therefore, the source of data collection is the set formed by the affected individuals themselves during the epidemiological outbreak of interest, which provides, for scientific research and discussions on combat policies, an important technical bias of the scenario and the engagement of people.

Although they consider that traditional surveillance cannot be substituted, the work presented by Mohanty, Chughtai \& Rabhi (2019) postulates that the use of mobile applications can be a useful complement for the early identification of epidemiological outbreaks. At work, a survey of mobile applications available at online stores and aimed at monitoring and controlling the impacts of diseases was carried out, in addition to providing data for specialists to carry out studies and establish strategies for action to combat diseases. The paper mentions 
IADIS International Journal on WWW/Internet

the lack of alternative applications aimed at the general public and available for free. Finally, it was concluded that there is enormous potential for new applications for the health sector, especially in the fight against diseases. The platform developed in the present project, in a complementary way, tries to stimulate the line of research related to collaborative collective health surveillance solutions, providing functionalities that allow the user to inform about their health and obtain assistance when necessary.

Data obtained through applications or other technological devices can also be used to feed a rich panel of information related not only to statistical information, but also to the feeding of geographic information subsystems (or Geographic Information System - GIS). Chiluba \& Dube (2020) focused their studies on reviewing and analyzing the geographical panels available online for mapping the outbreak of COVID-19, experienced in the year 2020. The panels analyzed used different strategies for geospatial representation of the impact of the disease. Among these strategies used by the analyzed tools, the following stand out: choroplethic, circular concentric maps; pie charts, buffer, overlay and those with animations. Unlike the work mentioned in the previous paragraph, it is dedicated to surveying analytical tools with a geospatial focus. By surveying existing tools, it establishes a qualitative comparison between them. This project differs in that, in addition to also having graphics from a GIS, the data source and collection methodology are well known and are available for access and plotting other graphics through a programmatic interface.

To broaden the context of the related works, this project aims to unite and explore the possibilities of each type of solution addressed in the works cited and referring to each stage of the surveillance process. Therefore, in the following section, the project and implementation of an alternative solution that integrates the stages of acquisition, storage, and analysis of health data in a single platform will be presented, assisting the teams that develop combat policies and those of research and development that act in the search for treatments and elaboration of forecasts and recommendations.

\section{PROPOSED ARCHITECTURE}

This work proposes the implementation of a platform capable of facilitating the visualization and decision making based on data on health outbreaks through information provided voluntarily by users. For this purpose, a set of software was developed, prepared to collect, process and display records in an easy way for the operators responsible for the solution.

This system consists of three different services: Mobile application, API (Application Programming Interface) and Control Panel, which provides and automates the analysis and aggregation of data. Each one, together with the strategies behind the choices, will be explained below, with the focus of this article concentrated on the added value to the work of health surveillance professionals through the third part of the platform described in Figure 1, the analysis and aggregation of data. It is also expected that the motivations and technical concepts to be addressed will serve as a guide, support, and inspiration for initiatives with a similar theme. 


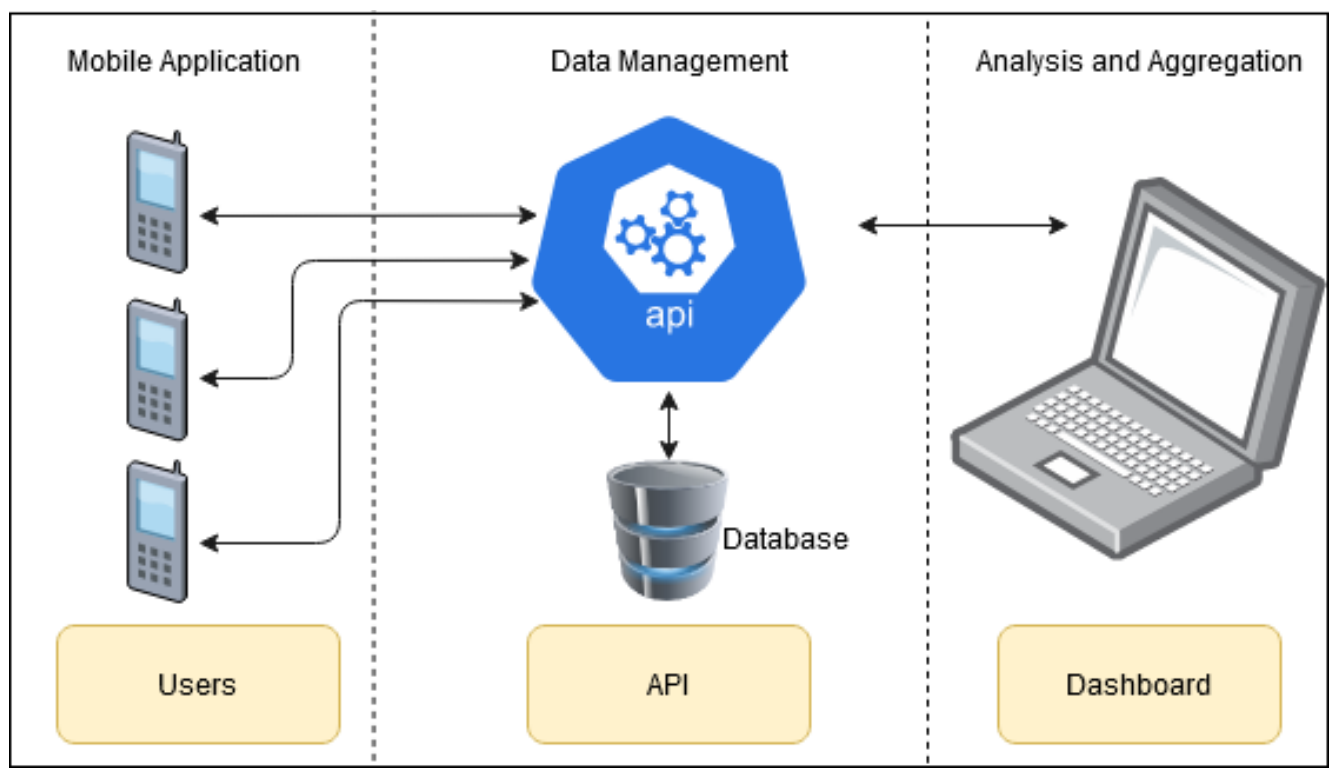

Figure 1. Platform structure separated into modules. Each module is responsible for a service

\subsection{Mobile Application}

The first concern of the project was related to the way of acquiring data and making it possible to receive alerts. This functionality will be explained later. Before any analysis, considering that it is a project that depends on data collected voluntarily, it is necessary to understand the strategy adopted for the interaction with users. In this case, it was necessary to choose a technology that was popular enough to reach any interested potential user, but that did not become so restrictive as to limit any benefits that could be reverted to the engaged community. Furthermore, it was necessary for this technology to be highly scalable and to allow access to information that does not have a trivial distribution, that is, that cannot be provided in a manual, simple and uncomplicated way by people, such as geographic location. In addition, it is also important to highlight that the choice of technology should favor the privacy of users, so that data such as location were collected only with explicit authorization from the user at the time of registration. The work of Caldas Filho et al. (2020) developed a platform with that addresses these issues.

This set of requirements led to the choice of mobile applications as a data collection point. Today's cell phones predominantly have Internet access and the possibility of fast and free installation of programs with a visual and interaction oriented to end users, in addition to being able to be installed without quantity restrictions, according to the native application paradigm (Huy \& vanThanh, 2012). In summary, the fact that each mobile device runs a program locally and consumes the services of a cloud application makes the user experience intuitive, independent, and highly scalable., These concepts are aligned with the type of architecture proposed for this work: the orientation services. Also, smartphones are part of the top of the list of most popular equipment in the world, according to forecasts and trends in Cisco (2020). Thus, the mobile module becomes a fundamental part of a project with ambitions for voluntary collaboration. In it, users should be able to register on the proposed platform and provide data 
IADIS International Journal on WWW/Internet

on their health status and profile (essential actions for the operation of data analysis). Also, the application will periodically and autonomously send the user's location, facilitating the entire surveillance process. This latter functionality also benefits greatly from the choice of mobile applications, because although it is not common for people to move around with geographic location trackers, smartphone devices provide not only this functionality, but also relevant processing power, the possibility of various automations, tasks in the background and very user-friendly interface.

It is important to highlight, regarding the location, that it is not featured on the panels. In order to maintain users' privacy, the visualization does not allow to know the identity of each person on the map, unless it is an operator with authorization (access level) for such purpose, which is intended, in an initial moment, to be quite restricted. The idea is that this possibility exists only for professionals who will handle alerts and monitor cases of infections. Access with less privileges, that is, that does not allow verification of personal data, would be used, for example, by researchers and administrators, who will not interact with the volunteers, but will use the global data for decision making and study.

\subsection{API}

As previously mentioned, the choice of a mobile application has the advantage of being highly scalable when the platform design is made based on the services division. However, it is important not only that mobile applications have this scalability, but that the programs that will request data and perform analyzes are also independent and have freedom of work. These concepts drive a project to differentiate between who provides and manages data and who enters and / or manipulates information. However, in applications available through the Internet, there are still considerations related to security, form and also the extent of each module's access to data. Also, the way in which these data are delivered can significantly burden software development precisely due to the fact that several operating systems from different platforms, with different representations of data (concept called "endianness", explained in Google Patents [2014]), are accessing and entering information on the same basis, without any protocol or algorithm to guarantee the isonomy of the representations. Finally, the choice of the interface for data raises other problems, including one related to the level of access by function: there is no need for each copy of the application to have full access to the database (just as a voluntary user should not have maximum level of access to the project data and not even have freedom of insertion in different formats than the application expects), precisely because each instance is executed in a private context (the user's cell phone). This enumeration of problems introduces the need for so-called API's.

An API defines a set of calls that can be used in an organized way by other programs to integrate different software modules. Each call is designed and defined by the programmers in such a way that the issues raised are obligatorily observed and treated for implementation:

1. Regarding form, programmers need to define the formatting and filtering of the data for each possible call to an API function before developing and making it available. This implies that the data is made available only according to the vision projected in the API interface.

2. Regarding the isonomic representation of data, an especially important problem in the context of Internet-dependent applications, an API is always developed based on a protocol defined by Industry standards. This protocol, among other purposes, must 
guarantee the correct conversion of data in a transparent manner for the final applications. In this case, the application layer protocol of the Internet's TCP / IP stack that defines the format of messages and calls is the Hypertext Transfer Protocol (HTTP), defined in Fielding \& Reschke (2014). Thus, regardless of the platform and software, there is a commitment to adapt the conversation to what governs the HTTP protocol, ensuring the equality of data regardless of platform.

3. Regarding the extent and level of access, each possible API call returns (or inserts) only the data defined during development. Therefore, each module, effectively, works in well-defined and consistent scopes of access to the database.

Therefore, to allow independence between the analysis and user interaction modules, to guarantee the good practices of a project separated by services with well-defined contexts and to provide high scalability in data management, an API based on the HTTP protocol with methods that regulate access to data and the inclusion of information in the bank in a consistent manner. For all the reasons and analyzes available, the design and development of an API is shown as a primary choice for any project based on voluntary interactions and data acquisition of end users through the Internet.

\subsection{Control Panel}

With the exclusive focus on visualization and generation of value for the data, a control panel (also called dashboard) was developed to aggregate the data that are sent by the users to the API and present it so that it is possible to either have a broad notion of the data set, or to follow changes almost at the same moment in which they occur. This is the module responsible for facilitating health surveillance and bringing health professionals closer to the end users.

This dashboard is divided into two sections, which are described as it follows.

\subsubsection{Registered Users}

In this part of the panel, it is possible to view, on a map or in a list, all users registered on the platform. A system of colors was used to visually distinguish the risk profile of users: those with green color are users under 50 years of age and who did not report any comorbidity; yellow represents those over the age of 50 or who have comorbidities; and red symbolizes those over 50 years old and, at the same time, have comorbidities. Comorbidities are understood as pre-existing health conditions that can add to other diseases and / or facilitate the infection of new diseases, such as obesity and hypertension, as defined in Valderas et al. (2009).

With the easy distinction between the different groups, it is simplified to direct the attention to users, especially considering the use of this application by health professionals, which will be discussed later.

It is also important to highlight that the proposed visualization has other features that go beyond the context of a single epidemic or disease. As an example, given the definition of comorbidity mentioned and the separation by age groups, it becomes possible to conduct studies to determine local factors that can stimulate the collective manifestation of these comorbidities and make decisions that directly affect people's health. 


\subsubsection{Alerts}

This tab provides easy viewing of alerts generated by platform users. The visualization can be in the form of a list or a map. A color system similar to that of registered users is also used, however, in this case the colors are arranged according to the reported symptom, not with the characteristics of the users. If only one of the symptoms common to various diseases is reported, such as headache, fever, or sneezing, for example, the green token is displayed. The yellow token is shown if two of these types of symptoms are reported, and the red token is a set of three or more symptoms or if the user reports critical symptoms that are closely related to COVID-19 disease, such as lack of taste, smell, or breathlessness.

The initial development of the application was motivated by the COVID-19 disease pandemic. However, the final application and project model is generic enough to be extended to the visualization of any data involving contagious diseases and cases related to health surveillance.

Another important point to be mentioned is that, as the user shares personal and health data with the application, it is possible to include health professionals in the surveillance process who perform more targeted and personal interventions for each user. This situation is especially relevant in cases of an epidemic, in which the monitoring of the infected is of paramount importance (Daumas et al. [2020] discusses the topic). Therefore, these professionals could, with the support of the platform, direct efforts, and assist, at a distance, people in situations of various vulnerabilities.

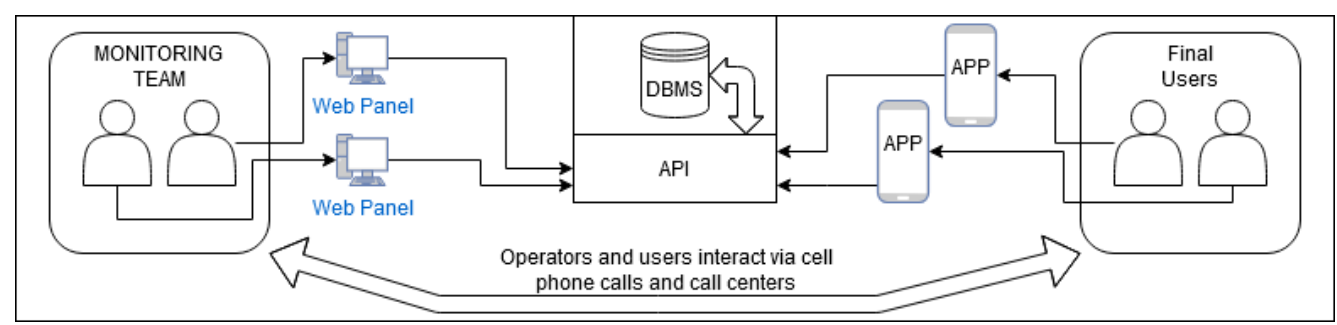

Figure 2. Path of data and features for the proposed platform

It is also interesting to note that the alerts issued by users contain their geographical location, facilitating the geographic monitoring of information by the professional who is on standby, so that it is possible to observe not only risk groups, but also heat maps. of the requests for help and the number of registered users, a very important vision for the health administration of a large city, for example, which can more efficiently re-manage ambulances and cross traffic data to reduce waiting time. Figure 2 shows a schematic of the intended use for the final solution in the context of a surveillance room.

\subsection{Role Based View}

This platform is the cornerstone of a business model based on the voluntary collaboration of users. In this context, it is extremely important to discuss, even if only briefly, how to minimize the effects of malicious registrations and increase the reliability of the acquired data. It turns out that it is not part of the scope of the proposed collaborative platform model to provide mechanisms to verify users. In addition, verifying the accuracy of an end user's information can be a very complex and costly task, requiring complex external queries and access to data that may not be public. 


\subsubsection{Trusted Third Parties}

Because of the difficulty in validating personal data, the project will make use of Trusted Third Parties (TTP), which is explained in Adams (2011). In this way, each user will have their identity validated by an external and trusted entity, and all users authenticated by that external entity will have access to the API resources and/or will be able to enter data on the platform. Figure 3 illustrates the idea of using authentication as a service provided by a third party.

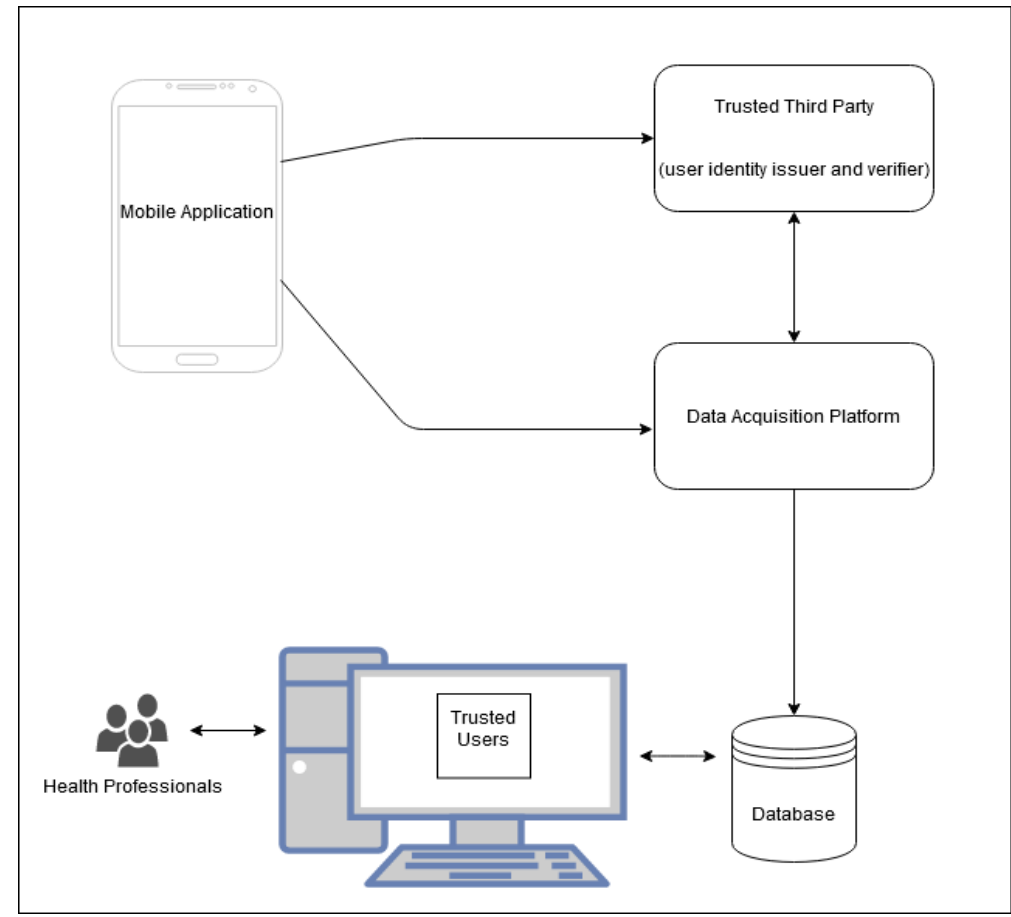

Figure 3. The authentication is a service provided by a third party. This ensures that the health professionals will see data coming from a verified and possibly unique source: the final user

In this way, it is possible to increase the reliability of the data since the authentication entity will be a trusted authority and the data owner. Take, as an example, the interaction of a user based on his general registration number, a unique and important registration code in Brazil. If the authentication entity is the Department of Public Safety, which is already responsible for granting and attesting the veracity of these registrations in the cited country, the platform will have greater guarantees that the end user is who he says he is and that the possibilities for duplication of information will be minimized. Another example could be a student and researcher authentication service. Once a university is integrated into the system, any students or researchers that are validated by the university will be accepted by the platform, and therefore the same guarantees of uniqueness of registration and data reliability will be automatically extended to the data acquired by the platform. Only questions related to data quality will remain, which depend much more on the experience and user engagement. 
IADIS International Journal on WWW/Internet

\subsubsection{Filtering Views}

It is not enough to just validate users and look for security guarantees in the registrations. It is still necessary to discuss how to adapt the system to data protection laws (a recent example can be seen in Chen [2020], where this topic is discussed in relation to the COVID-19 pandemic), separating the types of access, but without making the project a bureaucratic and not transparent platform. In other words, it is necessary to add another concept to be implemented: Role-based Access Control (RBAC), which is well explained in Shin \& Ahn (2000). In this methodology, applications and users should always have an authentication and association to an access level, or more precisely, to a role. In this project, the user will have his identity validated by an external and trusted entity, and all users authenticated by that external entity will have the same role, that is, the same level of access to API resources. Figure 4 shows the functioning concept of division in views based on the role of each user.

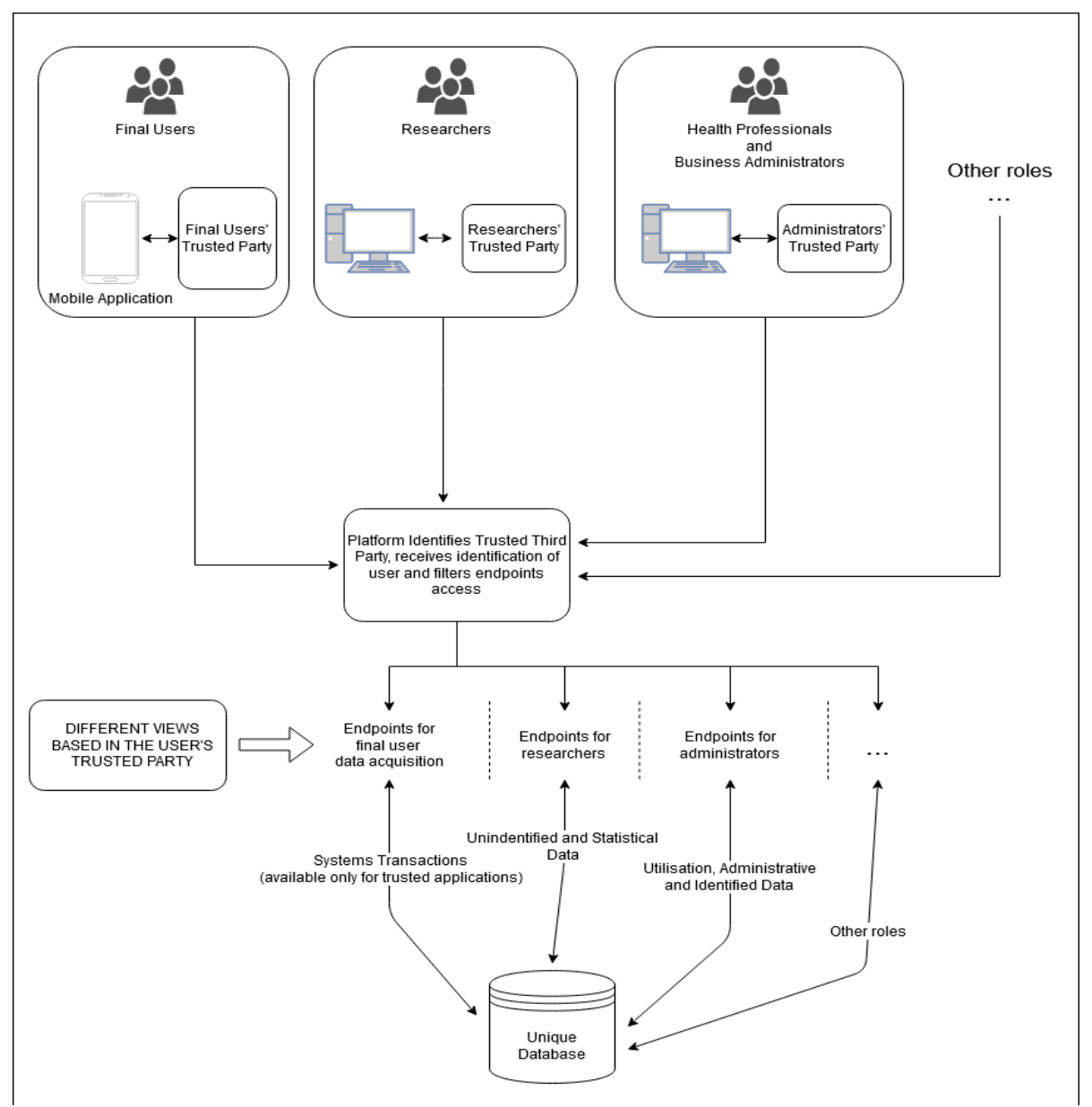

Figure 4. The API will be publicly accessible, but the users will see only the resources their organization is able to access. This means that a researcher asking for a list will use the same path that an administrator may use, but data will be sent without personal information and from an internal different endpoint 
The implementation of the division outlined in Figure 4 is important for the system to generate the greatest possible impact with the least bureaucracy. As it is a collaborative platform, most resources must be public, however, due to legal and security limitations, more complex personal data, and statistical calculations (which require more processing and generate more costs) would be part of a separate control. by users, but by organization and / or sectors. In addition, stimulating the need for partnership between different organizations is an important step towards generalizing the adoption of digital and security best practices. Therefore, a collaborative platform that implements RBAC associated with the use of TTP is a project that also fosters the digitization of essential services, collaboration between teams from different sectors with different interests, and the guarantee of good data security practices.

\section{TESTS AND RESULTS}

The system tests were carried out in an almost controlled environment: the system was put into production in a real use environment, but the group of users with access to the system was restricted. More specifically, 22 people applied to test the platform. This group was formed by students and professors at a university, being varied in relation to risk profiles and pre-existing health conditions.

With the software modules in production, users were asked to register on the platform with fictitious data and send health alerts at random times. Figure 5 shows the view of the georeferenced distribution of registered users with the respective colors according to the profile of each user. Tests were also carried out both to stress the view of alerts and to verify whether the quality of the requests on the map allowed adequate georeferenced visualization, as well as to validate the concept that a monitoring group could act in a concise, efficient, and timely manner. It is interesting to note that the accuracy of the location collected automatically could vary with the quality of the user's internet connection and with the Global Positioning System (GPS) signal, especially in confined and windowless locations. Also, the reliability of the alerts' arrival was satisfactory (as long as the user had access to the internet via cell phone), creating a queue which allowed operators to contact, by phone number, with each user who had placed the order (note it is noted that only operators in the service room who have access to the personal data of volunteers, professionals with other purposes have access only to the mass of uncharacterized data). Figure 6 shows the view that a monitoring team has of users asking for help through the application.

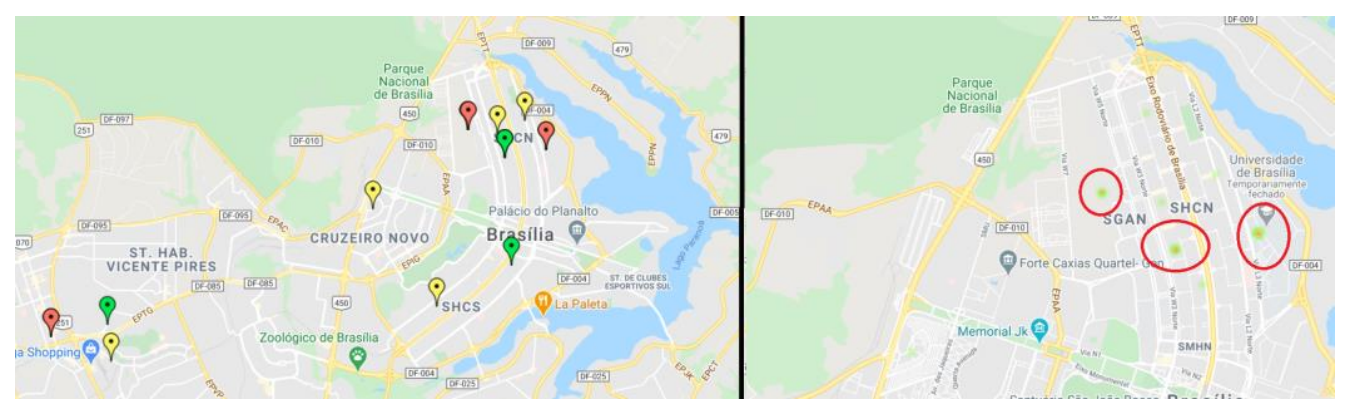

Figure 5. On the left, users are characterized by their profile. On the right, heat map with agglutination of alerts. The images are processed by the Google Maps API (Google Maps Platform, 2020) 
IADIS International Journal on WWW/Internet

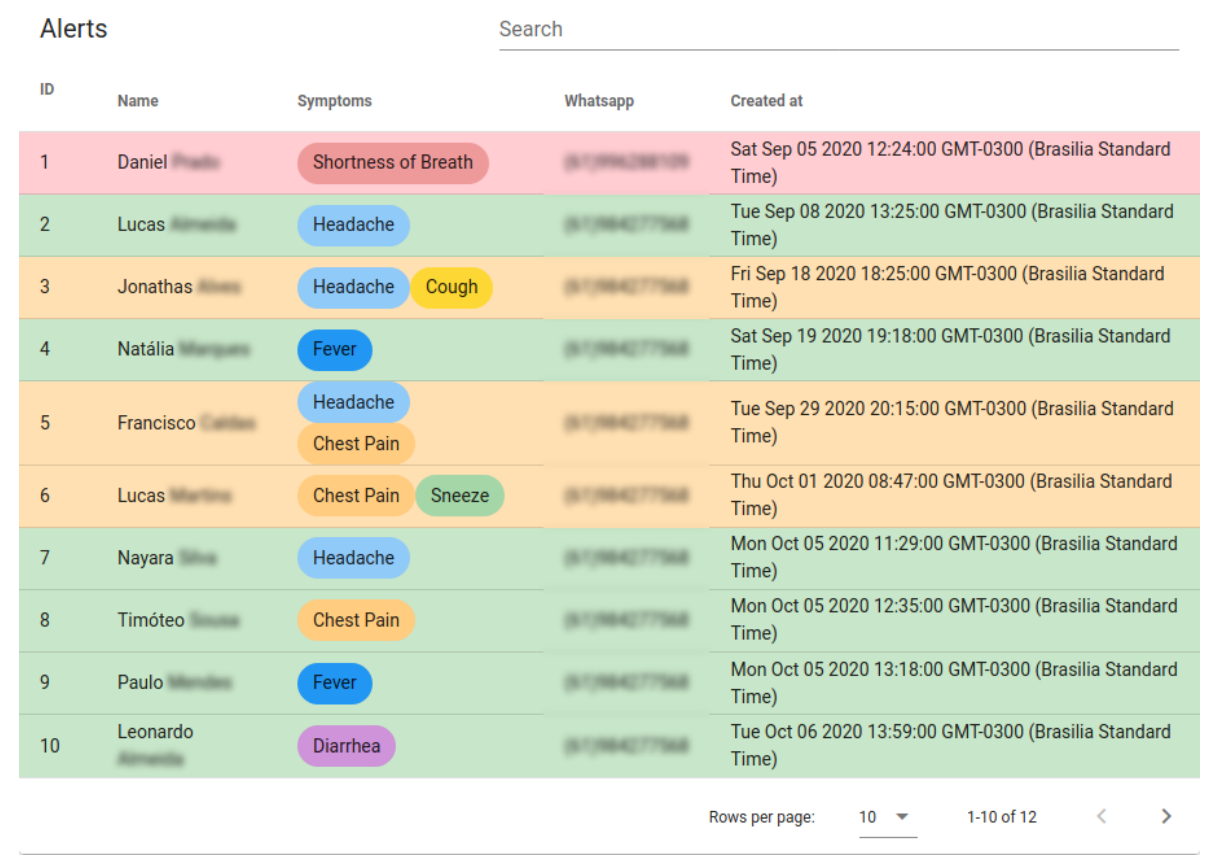

Figure 6. Panel with list of distress requests. All data is fictitious. However, the phone numbers were censored if they correspond to any real number. Last names were also censored where applicable

The value perceived by the aggregation of data and georeferenced visualization, as well as the power of collaboration by users and the ease in implementing a distress call service are quite evident when evaluating Figures 5 and 6 . The final product results in every logical apparatus necessary for the proper functioning of a collective health monitoring room, an initiative that becomes increasingly important the more relevant the health risks of large urban agglomerations become. Using the proposed system, a lean team can provide distress guidance services and deeply analyze the health of a large community.

Finally, Figure 7 shows the practical result of separating user roles. It is possible to see that the system and the user experience can be the same, but with different practical results. It is the adoption of these good practices that guarantees security and reduces bureaucracy for the use of the system. Each organization will have a role in the use and maintenance of the platform, which proves to be a very important data ecosystem for the integration of different initiatives. Public authorities, researchers, health professionals, private initiative and end users are now able to empower themselves with data and services without threatening the security and reliability of the model, in addition to complying with data protection laws. 


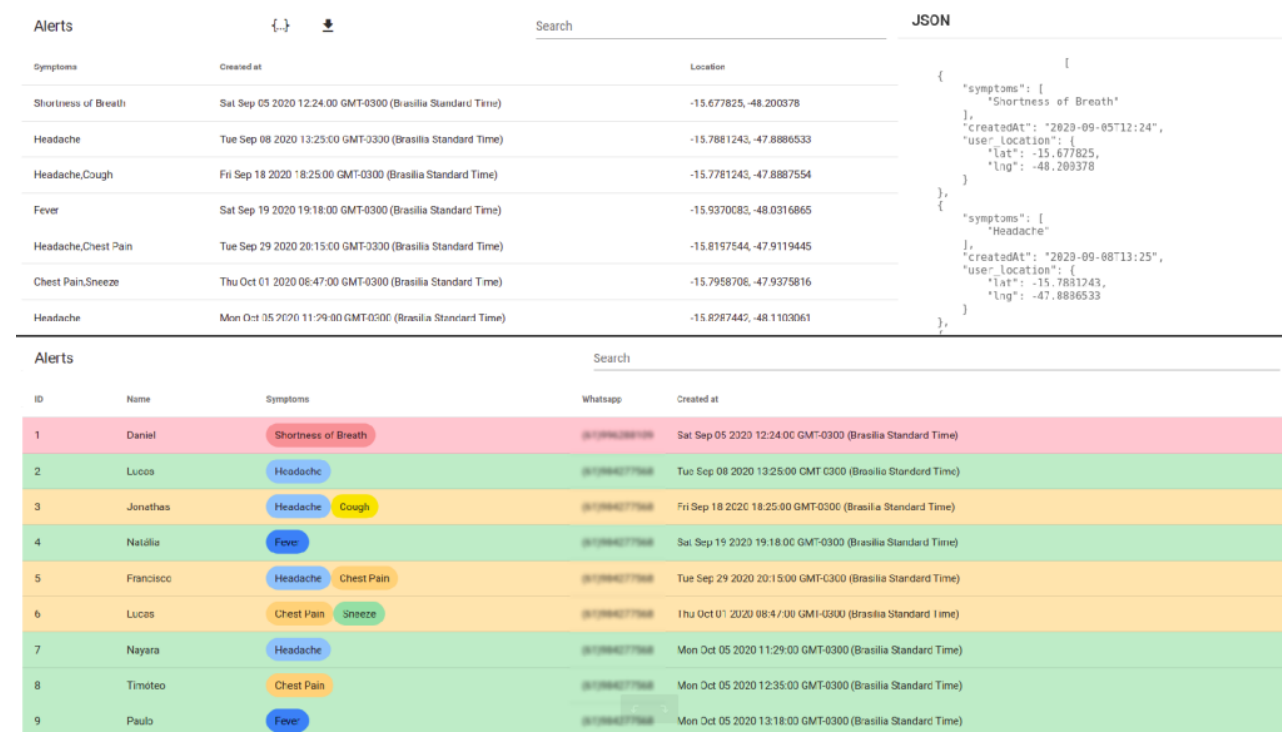

Figure 7. The top list shows the view of a researcher, who receives raw data without identification. The bottom list shows the view of an administrator, who does not see raw data in the list (such as geographic location) but has the view of personal data. The way the two interact with the platform is similar, but they produce different results

\section{CONCLUSION}

The COVID-19 disease pandemic suddenly appeared as a public health problem worldwide, having significantly impacted the lives of most of the population and demanding decisions in the most diverse segments of society, especially in health.

This context motivated this work, which presents the design and testing of a platform capable of supporting work forces inserted in scenarios not only of exposure to contagious diseases, such as an epidemic, but of general health incidents. The proposed solution works on two fronts: on the one hand, it is a channel that allows people to inform their health conditions and ask for help when necessary, and on the other, it allows authorities and health professionals to follow in almost real time and in a way collective health is facilitated. It is important to highlight that this work has as premise the collaboration of users in providing faithful data to allow the inference and conclusion of the contagion situation of the disease in the region.

The tool presented is a distributed system in which good design practices are considered in a way that allows the system modules to be scalable to be able to handle large volumes of data accessible from anywhere through the Internet. Therefore, the design and operation of the three modules built were presented in this work: the logic of the mobile application with a focus on end users, the control panel with a focus on the monitoring team and researchers and / or decision makers, and the API that allows communication between both contexts. 
The system was applied on an experimental basis in an academic community and it was possible to visually perceive places and people who needed more attention, as well as locate help requests made by users and have queues of notifications in chronological order and with differentiation of priority for better service management.

As a future work, it is envisaged the application of data analysis models, so that it is possible to make predictions about the evolution of the contagious disease in the monitored region. Another point to be explored is the possibility of integrating the data provided collaboratively by the system presented in this work with information obtained from other sources, for example, public health data provided by public health organizations and data collected on social networks. Still, the integration of the application with personal health monitoring devices can allow easy provision of health services for various situations, even of critical nature, such as the automatic request for help for victims of falls, heart attacks and vehicle collisions, for example.

\section{ACKNOWLEDGEMENT}

The authors would like to thank the support of the Brazilian research, development, and innovation agencies CNPq (Projects INCT SegCiber 465741/2014-2, PQ-2 312180/2019-5 and LargEWiN BRICS2017-591), CAPES (PROJECTS FORTE 23038.007604/2014-69 and PROBRAL 88887.144009/2017-00) and FAPDF (UIoT Projects 0193.001366/2016 and SSDDC 0193. 001365/2016), as well as the support of the LATITUDE/UnB Laboratory (SDN Project 23106. 099441/2016-43), cooperation with the Ministry of the Economy (TEDs DIPLA 005/2016 and ENAP 083/2016), the Office of Institutional Security of the Presidency of the Republic (TED 002/2017), the Attorney General's Office (TED 697,935/2019) and the Administrative Council for Economic Defense (TED 08700.000047/2019-14).

\section{REFERENCES}

Adams C. (2011). Trusted Third Party. In: van Tilborg H.C.A., Jajodia S. (eds) Encyclopedia of Cryptography and Security. Springer, Boston, MA. doi: 10.1007/978-1-4419-5906-5_98

Arias-Carrasco, R. et al., (2020). OUTBREAK: A user-friendly georeferencing online tool for disease surveillance. arXiv:2004.10490v1

Boulos, M. K. \& Geraghty, E., (2020). Geographical tracking and mapping of coronavirus disease COVID-19/severe acute respiratory syndrome coronavirus 2 (SARS-CoV-2) epidemic and associated events around the world: how 21st century GIS technologies are supporting the global fight against outbreaks and epidemics. In International Journal of Health Geographics, Vol. 19, No. 8. doi: 10.1186/s12942-020-00202-8

Caldas Filho, F. L. de; Almeida, L. de O.; Marques, N. A.; Mendes, P. H. F. C.; Cavalcante, A.; \& de Sousa Jr., R. T. (2020). "Design and Implementation of a Collaborative Platform to Assist the Academic Community with Problems Caused by the DO COVID-19 Pandemic [Projeto e Implementação de uma Plataforma Colaborativa para Auxiliar a Comunidade Acadêmica com Problemas Ocasionados pela Pandemia do COVID-19]". Conferências IADIS Ibero-Americanas WWW/Internet e Computação Aplicada, ISBN: 978-989-8704-25-2.

Chiluba, B. \& Dube, G., (2020). Descriptive Review of Epidemiological Geographic Mapping of Coronavirus Disease 2019 (COVID-19) on the Internet. In Biomedical and Biotechnology Research Journal (BBRJ), Vol. 4, No. 2, pp. 83-89. 


\section{DESIGN AND IMPLEMENTATION OF A COLLABORATIVE PLATFORM MODEL}

FOR EPIDEMIC AND COLLECTIVE HEALTH SERVICES

Cisco, (2020). Cisco Annual Internet Report (2018-2023) White Paper [Online]. Available at: https://www.cisco.com/c/en/us/solutions/collateral/executive-perspectives/annual-internetreport/white-paper-c11-741490.html (Accessed: 15 Aug 2020)

Consultor Juridico, (2020). Bruno Covas extends quarantine to June 15 in Sao Paulo [Bruno Covas estende quarentena para 15 de junho na capital paulista] [Online]. Available at: https://www.conjur.com.br/2020-mai-30/quarentena-estendida-15-junho-capital-paulista (Accessed: 15 Aug 2020)

Daumas, Regina P. et al., (2020). The role of primary care in the health care network in Brazil: limits and possibilities in coping with COVID-19 [O papel da atenção primária na rede de atenção à saúde no Brasil: limites e possibilidades no enfrentamento da COVID-19]. In Cad. Saúde Pública, Vol. 36, No. 6, pp. e00104120. doi: 10.1590/0102-311x00104120.

De Almeida, L.; do Prado, D. S.; Martins, L. M. C.; Silva, D. A.; Oliveira, J. A. \& de Sousa Jr., R. T., (2020). "Design and Implementation of a Data Visualization System Collaboratively Obtained [Projeto e Implementação de um Sistema de Visualização de Dados de Epidemias Obtidos Colaborativamente]", Conferências IADIS Ibero-Americanas WWW/Internet e Computação Aplicada , ISBN: 978-989-8704-25-2 .

Fielding, R., \& Reschke, J., (2014). Hypertext Transfer Protocol (HTTP/1.1): Semantics and Content (RFC 7231) [Online]. Internet Engineering Task Force (IETF). Available at https://tools.ietf.org/html/rfc7231 (Accessed: 15 Aug 2020)

Google, (2014). Google Maps Platform [Online]. Available at:_https://cloud.google.com/mapsplatform?hl=pt-br (Accessed: 15 Aug 2020)

Google, (2020). Multiple endianness compatibility - US Patent US10163467B2 [Online]. Available at: https://patents.google.com/patent/US10163467B2/en (Accessed: 15 Aug 2020)

Huy, N. P., \& vanThanh, D., (2012). Evaluation of mobile app paradigms. Proceedings of the 10th International Conference on Advances in Mobile Computing \& Multimedia (MoMM '12). Bali, Indonesia, pp. 25-30. doi: 10.1145/2428955.2428968.

Ienca, M., Vayena, E., (2020). On the responsible use of digital data to tackle the COVID-19 pandemic. In Nat Med, Vol. 26, pp. 463-464. doi: 10.1038/s41591-020-0832-5

Mohanty, B., Chughtai, A. \& Rabhi, F., (2019). Use of Mobile Apps for epidemic surveillance and response - availability and gaps. In Global Biosecurity, Vol. 1, No. 2, pp. 37-49. doi: 10.31646/gbio.39

Shin, M. E. and Ahn, G. -J., (2000). "UML-based representation of role-based access control," Proceedings IEEE 9th International Workshops on Enabling Technologies: Infrastructure for Collaborative Enterprises (WET ICE 2000), Gaithersburg, MD, USA, pp. 195-200. doi: 10.1109/ENABL.2000.883728.

Lai, Y., Yeung, W. \& Celi, L. A., (2020). Urban Intelligence for Pandemic Response: Viewpoint. In JMIR Public Health Surveill, Vol. 6, No. 2, pp. e18873. doi: 10.2196/18873

Santos, Alaneir de Fátima dos et al., (2017). Incorporation of Information and Communication Technologies and quality in primary health care in Brazil [Incorporação de Tecnologias de Informação e Comunicação e qualidade na atenção básica em saúde no Brasil]. In Cad. Saúde Pública, Vol. 33, No. 5, e00172815. doi: 10.1590/0102-311x00172815.

Valderas, J. M. et al., (2009). Defining comorbidity: implications for understanding health and health services. In the Annals of Family Medicine, Vol. 7, No. 4, pp. 357-363. DOI: 10.1370/afm.983

X. Chen, (2020). "Personal Privacy Protection in Big Data Environment Under the New Coronavirus Situation," 2020 IEEE 3rd International Conference on Information Systems and Computer Aided Education (ICISCAE), Dalian, China, 2020, pp. 651-653. doi: 10.1109/ICISCAE51034.2020.9236848.

World Health Organization - WHO (2020). Q\&A on coronaviruses (COVID-19) [Online]. Available at: https://www.who.int/emergencies/diseases/novel-coronavirus-2019/question-and-answers-hub/q-adetail/q-a-coronaviruses (Accessed: 15 Aug 2020) 